\title{
Additive Manufacturing Cases and a Vision for a Predictive Analytics and Additive Manufacturing Based Maintenance Business Model
}

\author{
Michele Urbani and Mikael Collan
}

\section{INTRODUCTION}

In the previous chapter we have seen that the literature on additive manufacturing business models can in broad strokes be divided into four different directions. To illustrate the real-world status quo with examples we discuss in this chapter two real-world cases in the context of using additive manufacturing technology in the production of medical prostheses and in the refurbishment of metal dies and discuss the business model aspects of both of these cases. The third part of the chapter is used to discuss a more

\footnotetext{
M. Urbani

Department of Industrial Engineering, University of Trento, Trento, Italy e-mail: michele.urbani@unitn.it

M. Collan $(\bowtie)$

LUT University, Lappeenranta, Finland e-mail: mikael.collan@lut.fi

(C) The Author(s) 2020

M. Collan, K.-E. Michelsen (eds.), Technical, Economic and Societal Effects of Manufacturing 4.0, https://doi.org/10.1007/978-3-030-46103-4_7
} 
visionary case, where additive manufacturing, together with predictive maintenance, allows one to rethink how the modern system of maintenance could work.

\section{Additive Manufacturing Used in Enhancing HeArt SURGery}

The use of additive manufacturing in healthcare applications has flourished in the past two decades [1] and the market share of additive manufacturing in this market was $\$ 6.1$ billion in 2016 [2]. The market share of additive manufacturing has caught a steady uptrend, a dramatic increase towards $\$ 21$ billion in 2020 is expected.

The improvement of old and the creation of new techniques for $3 \mathrm{D}$ printing, together with the development of new purpose-specific materials for the healthcare sector have made possible the deployment of a range of patient-specific applications [3]. For instance, the customization of healthcare products and services such as the realization of customized prosthesis, and the development of case-sized in-vitro models would not have, in many cases, been possible without the developments made in additive manufacturing technologies.

In this chapter we discuss the use of additive manufacturing in the treatment of heart disease from the points of view of the medical procedure involved and the technical solution that additive manufacturing can offer. Cardiovascular diseases (CVD), which are the focus of this realworld example, are a top ranked cause of death on a global level. All in all they were responsible for 17.9 million of deaths in 2015, with almost a $50 \%$ increase from 1990 [4]. The added value of additive manufacturing in the process is discussed. The chapter is based on the real-world collaboration between the Department of Industrial Engineering of the University of Trento (Italy) and the Azienda Sanitaria per i Servizi Sanitari (public company in charge of the provision of healthcare services) of the autonomous Province of Trento (Italy).

\section{Atrial Fibrillation: The Condition and the Surgical Intervention}

Persons who suffer from a condition known as the non-valvular atrial fibrillation (AF) may be subject to the occurrence of blood clots, which 
after being formed within the left atrial appendage (LAA) can enter the blood stream and cause a stroke, or other vascular complications. Many patients are regularly treated with oral anticoagulants, which are aimed at preventing the formation of the blood clots. Unfortunately, this therapy is not always possible, since some individuals have low tolerance of anticoagulants or the risk of bleeding problems caused by the anticoagulants is too high.

An alternative to using anticoagulants is to permanently seal off the LAA - the procedure does not solve the problem with AF, but it helps to prevent blood clot formation through the closure of the LAA.

The surgical intervention in question is called left atrial appendage occlusion, also known as the left atrial appendage closure. It is a noninvasive non open-body surgical intervention. There are a number of techniques that can be used to occlude the LAA, one of the most recently introduced practices consists of placing an implant via trans-esophageal echo guidance. The purpose of the implant is to ensure the closure of the LAA and to impede the flow of blood. The intervention is carried out under general anesthesia and is similar to the implantation of a stent.

Since the geometry of the human heart is different for each patient, the size and shape of the implants to be installed are of fundamental importance. In this context, the decision-maker is the surgeon, who bears the responsibility for the outcome of the surgery. The standard process to treat atrial appendage occlusion begins with a computed tomography (CT) scan of a patient's chest. This allows the doctor to create a rough estimate of the shape and size of the implants that will be implanted. CT is an effective tool and provides a set of cross sectional images of the scanned body along the sagittal plane. The set of images can be processed via a $3 \mathrm{D}$-software and a model of the heart can be created - this allows the patient's LAA to be inspected along the desired direction and gives the surgeon a better basis for decision-making. While the CT scan images and the $3 \mathrm{D}$ model are helpful, it remains difficult to foresee the practical difficulties that may arise during the operation.

In the current practice, implants are mass-produced according to standardized shapes and sizes, which forces the surgeon to choose from among a set of possible implant alternatives. With the aid of the CT scan, the doctor can narrow down the set of implants that could fit the heart of a given patient, but there is no full-proof way to in advance identify the right implant alternative. 
In practice the fit of the implant is directly tested on the patient during the surgery. Once the right dimensions have been found and the final decision on the implant made, the implant is implanted. The regular procedure foresees that patients can be released after a 24 hour recovery which is typically followed by a 45 day anticoagulant treatment. The success of the intervention cannot be determined immediately after the execution, due to the time required by the human body to adapt to the presence of the implant, in fact the verification takes place during the weeks following the surgery through periodic checks

In case the procedure fails, the operation is typically repeated and the implant is replaced with a better fitting one, thus subjecting the patient to a second intervention. The failure of the process may have severe consequences for the patient, such as pericardial effusion, incomplete LAA closure, dislodgement of the device, and other risks related to catheter-based techniques [5].

\section{Enhancing the Procedure with the Help of Additive Manufacturing Technology}

Additive manufacturing can be used to reduce the uncertainty connected to making the selection of the implant used and to assist in planning the surgery ex-ante. Contrary to what the reader might expect, the target of using additive manufacturing in this context is not the creation of a tailormade implant, but finding a best fitting mass produced implant. While it is logical to expect that once a 3D model of the patient's heart is available an implant of the right size and shape could be additively manufactured, however the low cost of the mass-produced implant and their availability on the market does not make it profitable to print them. Instead a real-size copy of the patient's heart is printed, based on the 3D model obtained from the CT scan. This allows the surgeon to test fit of the (different) mass-produced implants. The process is relatively low cost and provides the advantage of being able to perform pre-surgery testing of fit and by reducing uncertainty increases the chances of a successful operation. The life-size printed model of the heart also allows the surgeon to study the heart and the execution without any pressure, which may prevent practical difficulties during surgery to become overwhelming.

The 3D model produced from the CT images is practically print-ready, no processing by a human operator is needed. A software tool provided by the $3 \mathrm{D}$ printer supplier analyses the model and schedules the work for the 
printer and adds the print-support structures needed, before the model can be sent to the machine for printing. The printing technology used is stereolitography and it is one of the oldest $3 \mathrm{D}$ printing technologies. Patented in the United States in 1986, stereolitography uses a generic photopolymer-specifically a thermosetting resin monomer to build the printed object layer-by-layer on a build platform. Each layer is solidified by a UV laser beam that moves all over the cross-section and is used to solidify the resin. Once a layer has been completed the build platform is lowered and a new layer of material is injected on the cross section-before the new layer is solid, the excess liquid resin is wiped out in order to obtain the right layer-thickness; this also ensures that the surface is even enough for the application of the forthcoming layers. Finally, the printed heart must be finalized by removing the printing supports mechanically (by hand).

The material used (resin) does not have to be bio-compatible as it is not used in contact with the human organs and it can be chosen based on needed mechanical properties of which elasticity is the most important in being able to replicate human tissue-like behaviour during the testing and surgery planning phase. The resin typically used is the softest provided by the supplier and has a Shore-hardness of 50A. Compared to many other photopolymers for $3 \mathrm{D}$ printing, the substance is very elastic and it can reach $160 \%$ of elongation before breaking. The printing process including the post-treatment of the polymer takes a few hours depending on the complexity of the printed object. The short printing-time together with a good surface finish of the end product, print resolution is up to 100 microns, make stereolitography a suitable technology for this application.

\section{The Business Model Perspective}

The application of additive manufacturing described above presents features that are also found in previous literature [6] as hallmarks of successful implementation of additive manufacturing in practice-low production volume, customization of the product, on-demand production, the availability of the (3D) model, and the (modest) cost of the printing equipment.

The cost of a suitable stereolitographic printer ranges between $€ 3000$ and $€ 5000$, which in the context of the healthcare sector is a rather affordable investment for most medium and large hospitals, if knowledgeable personnel is already in place. The cost of the resin monomer is approximately $200 € / 1$, which translates to a material cost of some tens of euros 
per printed heart. In the context of this case, the region of Trentino-Alto Adige in the North of Italy, the yearly number of operations of the described type is less than one hundred; the region has approximately one million inhabitants.

The low volume of printed hearts produced may nevertheless make it unreasonable for a single hospital to acquire a printer, in which case renting the printing capacity could be a more cost-effective solution. Many research-centres and universities are likely to own a suitable printer and a partnership between hospitals and local research-institutes are a logical way to create win-win partnerships around this theme. More importantly, research-institutes typically have the manpower and expertise to manage the printing process. Starting from a scratch, the time required to acquire the knowledge to manage a polymeric printing process is reasonablewith a few weeks of training, a person is able to manage the whole process.

To summarize, in the present context it does not make sense to talk about a proper business model for this additive manufacturing application, however there is a clear benefit to using additive manufacturing to enhance the treatment of atrial fibrillation. On a scale of multiple hospitals and some thousands of printed hearts on an annual basis there might be a profitable niche for a specialized AM manufacturer. There is always business relevance in being able to provide superior techniques for medical purposes that lower the risks of surgical interventions-this refers to what has previously been characterized as incremental change in adopting additive manufacturing [7].

\section{Refurbishing Metal Dies with 3D-Printing}

In the context of manufacturing, it is very common to have processes that require a physical contact between a manufacturing machine and the processed product. When the contact is made in order to modify the shape of the processed item it is inevitable that the part of the machine that makes the contact, the so called die, will be subject to wear. The die can be made of different materials, but here we concentrate on metal dies. Many different surface treatments and improvements in the grade of the base material for dies have been designed in order to limit the effects of wear on metallic dies. While the metals used are hard, on the long run it is inevitable that a die looses nominal geometry, or the surface of the die becomes defective.

The more severe defects are typically surface cracks, sub-surface cracks, and the loss of material from the surface of the die. Generic loss of the 
nominal geometry is a type example of less severe defects that occurs with time in the most highly stressed areas of the die. Change of die geometry is a common cause of low quality in the end product. The general picture is that wear causes the end product of the process involving the die to decrease in quality and at a point when the quality-decrease at a limit to acceptable level the die used must be replaced or refurbished.

Due to the above issues, some metallic parts of manufacturing systems must in practice constantly be monitored through the inspection of the quality of the finished items, or through the inspection of the status of the parts themselves. Control performed by way of inspecting finished items is typically based on comparing the produced parts to the design specifications and when the design tolerances are no longer satisfied action must be taken. There are opportunities for predictive maintenance in these casesminimizing the number of unsatisficing end products is a cost issue.

Maintenance of a system component most often requires stopping the machine, which causes a loss in productivity - this is why the need to repair machines quickly and efficiently is as old as the manufacturing industry itself. Here we concentrate on the maintenance of metal dies used in manufacturing and especially on the refurbishing of metal dies by way of additive manufacturing technologies.

\section{Refurbishing Metal Dies}

The current practice of refurbishing metallic dies is based on manual labour. After the defective die has been found via a visual inspection of the production line, it is removed and prepared for maintenance. If the defect is a surface-crack the damaged region of the die will typically consist of an irregular surface on which work cannot be done-machining is first done to remove irregular surfaces, this is done by an operator by hand with a milling machine. In this task the die must be carefully placed in the milling machine and the position of the die and the machine must be calibrated. The end-result is a cavity with a smooth surface.

The cavity is then filled with a suitable filler-metal, typically a manual electro-welding process is used. After filling the cavity the die undergoes re-machining so that the original required (nominal) geometry is reobtained. This means that a milling machine is again used, after loading the die and calibration of the position on the machine. An error in the positioning of the die in the milling machine will compromise the success of the whole operation. While the manual refurbishing of the die is 
relatively inexpensive the risks related to the positioning and calibration of the die in the milling machine remains a problem. The main phases of dierefurbishment are visible in Fig. 1.

\section{The Hybrid Manufacturing Approach to Refurbishing Metal Dies}

Thanks to recent developments in the field of manufacturing equipment development, new bybrid equipment has become available. A hybrid manufacturing workstation embeds two, or more, manufacturing technologies within it. Typically this means that the elements of both additive and subtractive manufacturing are present in the same system. The clear advantage of a hybrid workstation is that as it is able to perform a large number of operations the set-up costs are typically lower. Specifically, only one instance of pre-processing (including calibration) is needed if the hybrid workstation is able to perform an operation, for which multiple machines are otherwise needed-this may dramatically reduce the time consumption as well as the risks related to pre-processing. Hybrid work stations are operated by software designed specifically for these machines. The downside of modern hybrid machines is their relatively high cost.

The hybrid workstation used in refurbishing dies is a DMG Mori Lasertec 65-the workstation integrates laser-deposition melting technology with a 5 -axis milling station. The station is able to automatically change between the laser- and the milling-heads. Limitations that the workstation has have to do with the volume and the weight of the workedon parts $(\varnothing 500 \mathrm{~mm} \times 400 \mathrm{~mm} ; 600 \mathrm{~kg}$ ) - this kind of limitations are "real" in terms of the workstation not being able to handle larger and heavier objects; as technology is developed further these limitations are slowly relaxed, but the limitations mentioned are on a "good modern

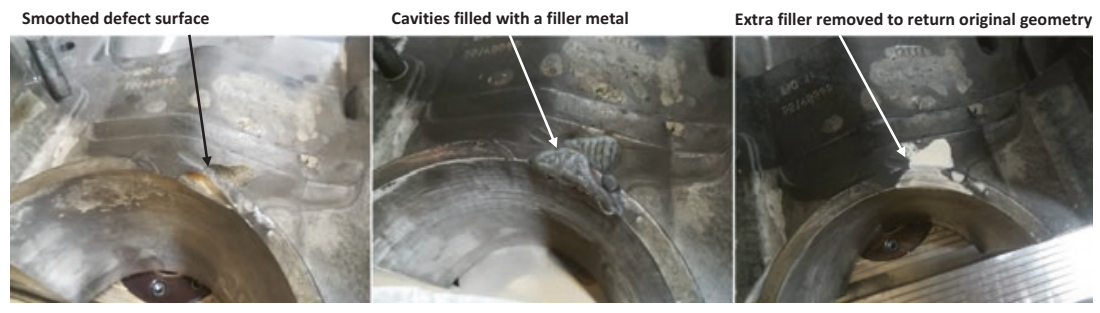

Fig. 1 The three main phases of refurbishing metal dies. (Original photos Matteo Perini) 
level". In heavy industry the die component size can still be too large to fit into hybrid workstations for quite a while. The workstation is able to handle various metals and alloys that include stainless steel, nickel-based alloys (Inconel 625, 718), tungsten carbide matrix materials, bronze and brass alloys, chrome-cobalt-molybdenum alloys, stellite, and tool-steel. The CAM/CAD software used is the Siemens NX.

The process of refurbishing dies with the hybrid workstation begins with the setting up and calibration of the damaged die in the workstation and is followed by a 3D-scan and the subsequent construction of a virtual model of the damaged die. A separate software is used for 3D-scanning. The accuracy of the virtual model depends on the resolution of the scanner. The accuracy is a relevant issue, as the more accurate the model is the more accurately it can be decided, which parts of the damaged areas need to be removed-typically the more is removed the more needs to be added later on. If the metal alloy used is very expensive the ability to use less material may have a positive effect on the total cost.

After the decision has been taken, the virtual model is compared to a model of the original (nominal) geometry of the die. With the original model and the virtual model of the damaged die it is possible to obtain the difference between the two and "instruct" the workstation to reconstruct the nominal geometry. The accurate reproduction of the original topology by additively filling the cavities to be repaired is the result of a focused research project at the University of Trento that developed a new method [8] and supporting software that translates the topological difference to a set of machine-understandable instructions that the CAM software is able to read.

The laser deposition melting solution used allows a homogenous distribution of metallic powders, which occurs under the protection of a shield gas that protects the process from oxidation. The system construct is such that a separate work chamber with a controlled environment is not needed-this makes the process faster that is typically the case. It must be observed that the die typically consists of two metallic parts - the part that can be called a "saddle" that is connected to the machine and the "contact part" that is made of a harder metal and that is attached to the saddle and that is the part of the die that is in contact with the produced parts. The actual additive manufacturing procedure is divided into three layers, where the first layer is the (material of the saddle part of the) refurbished die, the second layer is called a dilution zone and it is a mixed material made partly of the original die saddle metal and partly of the filler metal (contact part), 
and the third layer is fully made of the filler metal. This three-part procedure is able to produce a very durable non-porous and crack-free metallic solid-the refurbished die can be said to be "as good as new", which is the best possible end-result.

\section{The Business Model Perspective}

Similar to the heart implant example above, also in the case of die refurbishing the uniqueness of the procedure and the product are key elements - that is, the unique faults in the dies offer a possibility for additive manufacturing to be competitive. Furthermore, as the dies are typically constructed of two metals the reconstruction process of a die is not simple and the ability to refurbish dies to "as good as new" state requires handling high product complexity in an efficient way, something that is possible with the hybrid workstations presented above. This also means that if dies are refurbished en masse that there is always an element of customization to the work-identifying the refurbishing procedure for the various kinds of faults allows something that resembles mass customization. If a relatively expensive hybrid workstation is acquired with a profit in mind it is clear that the workstation should have an as high as possible rate of utilization. This means that there should be a number of different dies (and other parts) for the refurbishing of which the processes should be wellknown and ready.

In such a case, even a single hybrid workstation could act as a part of a number of maintenance supply chains and in essence function as a machineas-a-service (MaaS). The workstation could be purchased through a leasing contract by the customers, who pay an annual fee for the use of the machine, or the machine is acquired by one "player" who then sells the capacity of the machine to others - there are many possible types of ways to organize the availability and the sale of the capacity of the workstation. In the case of the refurbishing metal dies the workstation can, e.g., be a part of a die maintenance chain that consists of predictive maintenance system in place at one or several manufacturing facilities that use(s) metal dies and that is able to refurbish-on-demand. Persona et al. [5] write about maintenance outsourcing and the resulting effects on supply chain organization.

For a manufacturing company the number of dies that need refurbishing on recurring basis must be large enough to warrant the relatively high costs of acquiring and operating a working station, which indicates that 
such a move would make sense only for large-enough operations. If a workstation is present on-site any logistics costs are reduced-this may have a marginal positive effect on the cost side, however the potential to shorten downtimes with on-site refurbishing may have a more remarkable positive effect. In a broader perspective the adoption of additive manufacturing technologies must be regarded as a strategic choice for a firm. Purchasing AM capable machinery, such as the hybrid workstation, an organization makes a long-term commitment into a new technology, which not only includes the cost of equipment, but demands the acquisition of the related human talent. We refer the interested reader to Weller et al. [9] for additive manufacturing cases in maintenance applications.

One option in this space is to outsource the maintenance of the metal dies and buy "dies as a service". There are specialized firms that exclusively sell industrial maintenance capacity and in a sense machine availabilitytypically in these cases the production facilities belong to customer (manufacturing company) and the service provider is in charge of their good functioning. This option will be discussed more in the remaining part of this chapter, where disruptive maintenance-related business models that rely on digitalization and excellence in additive manufacturing are presented.

\section{Predictive Maintenance and Additive Manufacturing: Joint Business Model}

Broadly speaking, predictive maintenance is the practice of scheduling and performing maintenance in a way that predicts failures and is hence able to contribute to minimizing production downtimes, maximizing component lifetimes, and to minimizing maintenance costs, we refer the interested reader to see [10]. The indirect benefits that predictive maintenance brings include the potential to use maintenance resources more efficiently, the ability to carry a lower inventory of spare parts, and the important ability to make "tougher" production-related promises to customers. These benefits accrue to both the owner of the maintained system and to the organization responsible for the maintenance that can also be the same organization. The ability to make maintenance more efficient is a source of lasting competitive advantage.

Predictive maintenance is winning ground in manufacturing (and elsewhere) due to the instrumentation of manufacturing equipment that 
allows automating the collection of condition data. Based on the data collected predictive models can be tuned in a way that enables the accurate prediction of the timing of equipment failures and the construction of smart maintenance schedules.

Different architectures for predictive systems exist, perhaps the most prevalent at the time of writing are "monitoring-based" systems that track deviations in the system captured by sensors and alert as they appear. Different types of deviations may have different types of "fingerprints" and known the tell-tale signs of a deviation allows the correct classification of the deviation and the correct prediction of an incoming fault. These systems are evolving in the sense that their ability to identify failures becomes better with time as more and more data is accumulated and the patterns that distinguish the different failures become better known. In essence these systems utilize "machine learning".

Smart means in this context also the ability select a good maintenance policy that keeps the level of unexpected component failures (and stoppages) at an acceptable level. Smart increasingly means also being able to answer to more difficult questions such as: "once equipment is shut down for maintenance, what else than only the minimum necessary maintenance should one do?"-questions of this type and finding answers to them is difficult and requires system-size modeling for maintenance optimization.

Maintenance optimization work typically includes the modeling of the maintained system and the individual maintainable components (including modeling the wear and tear) and the optimization of the system maintenance based on the model. Bundling maintenance actions in an optimal way is a complex optimization problem and requires considerable computing power and good modeling. So far the typical target of maintenance optimization has been a single system, however, it is clear that the optimization of multiple systems simultaneously offers added benefits. If issues such as workforce scheduling are also taken into consideration in the optimization the complexity of the optimizable problems increases, but on the other hand so do the potential rewards.

One can without a doubt make the claim that the sophistication needed in maintenance optimization is at par with the sophistication needed in the rest of the Manufacturing 4.0 paradigm-someone might even go as far as to say that smart maintenance can be seen as a part of the paradigm when the maintenance context is manufacturing. 


\section{Predictive Maintenance Based Business Model for Additive Manufacturing}

What makes predictive maintenance different from "typical maintenance" is that due to the instrumentation in place even ad hoc (un-expected) failures can be predicted-in other words there is typically sufficient time to react between acknowledging that a component is about to malfunction and the actual time the component breaks. This period of grace that results from the predictability of faults can be utilized to render the manufacturing operation more efficient by determining the optimal maintenance actions that are performed, when the component that is known to malfunction is changed and by making ready the preparations for the said actions to be performed-including procuring the needed components that need to be exchanged. In this context the procuring the components is the key issue, because the new replacement components can be taken from a (local) storage if they are available, brought to the failing machine from a storage or production location further away, or produced on-site (or near) by additive manufacturing.

Implications of enforcing and making stronger the connection between predictive maintenance and additive manufacturing are quite remarkable-in cases where a failing component, or a spare-part, can be manufactured in time and on-demand for the maintenance action to take place, there may be and there most likely are savings to be made. In the case, where the alternative is transporting a spare-part from far away, which is by far not unheard of. If the on-site (additive) manufacturing of spare parts becomes the trend, the logistics of spare-parts becomes less of an issue and in fact the "logistics middleman" can be even completely cut out. Spare parts logistics are replaced by the logistics of the much less expensive and non time-critical logistics of the materials needed to produce the spare part(s) on location and the digital logistics of the information needed to print the spare part.

One can observe that also the need for the storage of spare parts is diminished as only spares that cannot be printed on demand must be stored-as time passes it can be expected that the selection of materials available for additive manufacturing grows wider and the quantity of nonprintable parts grows smaller. Generalizing and perhaps being slightly polemic one may surmise that if there is a revolution by additive manufacturing, then it surely must also be a revolution in logistics. As logistics costs are not insignificant there is a clear potential for savings immediately, 
when the cost of production of spare parts by way of additive manufacturing become competitive. We refer the reader interested in the supply chain effects of additive manufacturing to see [11].

The above described predictive maintenance-additive manufacturing symbiosis requires quite seamless informational collaboration between the activities of maintenance (and operation of the maintained equipment), which typically require full knowledge of the design of the said machine, and of the parts production for the machine. In other words, the collaboration of a number of stakeholders in the process is necessary in a way that is very fast, and in the best case automatic.

Automation means that there is a need for a standard system level "rules of play" that govern the informational and trade exchanges taking place within the system, including a joint understanding and pre-acceptance of the involved costs. With the costs we refer, among other things, to the cost of the rights of use of the "recipe" or the digital plans required to print the spare parts, whose IPR typically resides with the original equipment manufacturer.

The fact that a number of things need to be pre-planned and preaccepted creates a great a "natural" hurdle, when (multiple) separate organizations need to reach consensus - it is therefore likely that the first working systemic solutions that incorporate these technologies in the way envisioned above are formed by actors that already control the different steps of the maintenance and spare-parts production whole and are therefore able to benefit from any and all efficiency increases and cost savings related to process changes.

\section{Blueprint for a Vision}

Instrumented equipment is able to digitally transmit real-time information about the condition of perishable parts to what is called "predictive maintenance optimization system" in Fig. 2. The idea is that a sophisticated maintenance analytics system is able to utilize data coming from the sensors located in the production equipment (\#1 in Fig. 2), to create results by utilizing modern condition-based maintenance and predictive maintenance models (for ad-hoc failures), and to use the results as input in a sophisticated maintenance action optimization. Modern optimization systems are able to intelligently group maintenance actions to realize potential cost savings from performing multiple maintenance actions simultaneously. Putting smart maintenance planning automatically into 


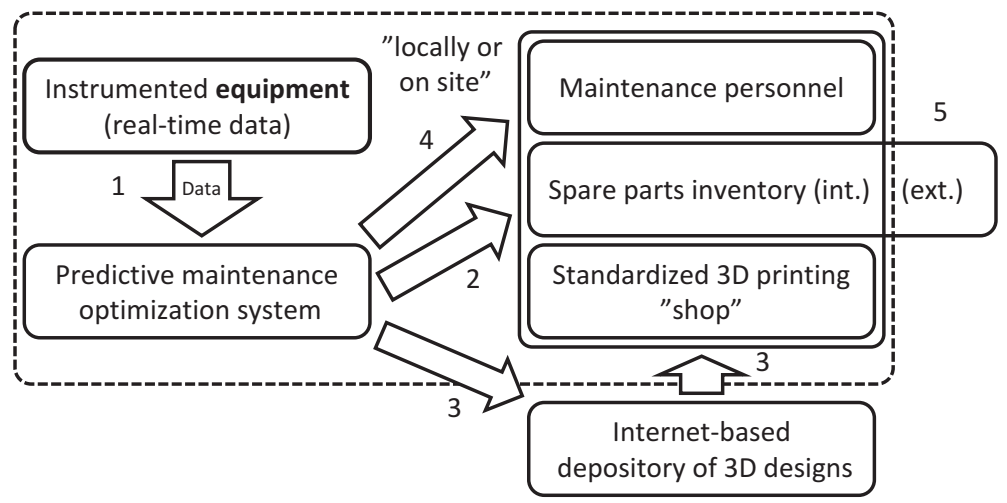

Fig. 2 Additive manufacturing "3D printing shop" as a part of a predictive maintenance oriented maintenance ecosystem

action means that the optimization system has the ability to check the local spare parts inventory for existing parts that are needed (\#2 in Fig. 2), to order needed designs from the on-line OEM depository of 3D designs for the parts that need to be printed, and reserving time slots for printing from the 3D printing facility (\#3 in Fig. 2).

The maintenance optimization system can also reserve (and in some cases even optimize) the maintenance personnel resources needed and schedule the actual maintenance (\#4 in Fig. 2). In contrast to the traditional model, where the locally non-available parts would be searched for and ordered by the automatic system and then shipped to the location from an external warehouse possibly on another continent (\#5 in Fig. 2) the additive manufacturing based model can allow for all physical actions to be performed on location. It is clear that a hybrid of the "old and the new" is a state that may be in place for a long time and where the smart optimization system is ultimately able to decide whether to order spare parts from an external warehouse or from a local 3D-printing facility based on minimizing a cost function that may include, e.g., time penalties.

In an ideal world the optimization system is able to create a circumstance, where the costs are minimized, optimal amount of maintenance is carried out, parts are ready just-in-time, and personnel resources are optimized. The driving forces behind reaching this kind of a state are the development of digital instrumentation in equipment (IoT), development of smart predictive maintenance systems that are coupled with advanced 
maintenance optimization systems that are digitally connected to resource management systems. The vision presented includes an Internet-based depository of $3 \mathrm{D}$ designs as a component-such depositories already exist for hobbyist designs, as of yet serious B2B depositories have not emerged.

Many different kinds of business model possibilities exist within the vision, the envisioned whole can be realized within the "realm" of a single actor, or by way of collaboration of specialized single actors.

\section{Conclusions}

This chapter has concentrated on presenting two real-world cases of how additive manufacturing can be used to enhance existing processes that otherwise demand precision manual labor and/or cannot be performed as well. Both of the real-world cases show that there is potential in additive manufacturing in places, where sophisticated tailoring of what is done is required, and where precision is a key factor. In both the cases the business model aspects had not been fully explored due to the exploratory and piloting nature of the activities performed, but it remains quite clear that with a high-enough demand for the presented activities there is a profitable business case to be made. If a specialized know-how is created around an additive manufacturing resource, the resource can be leveraged to service multiple different clients. It must be observed that in the same way as with any production technology, if the utilization rate of the equipment used is low the chances of reaching profitability remain a challenge-the laws of production economics do not change.

There seems to be a place for visioning additive manufacturing based business models that combine additive manufacturing with other technologies, such as predictive maintenance, as presented in this chapter. The ability of additive manufacturing to deliver on-demand is an important factor from the point of view of efficiency gains it is able to bring to the business of which additive manufacturing is a part of. When coupled with "control" technology that is able to make just-in-time orders and to optimize processes the ability to produce just-in-time can be exploited effectively. The prospect of locally manufacturing with additive manufacturing technologies through a global web of digital information is an interesting one and puts pressure on mass-production and long-haul logistics based business models.

Industry-grade $3 \mathrm{D}$-printers can be thought of as platform investments that service more than on client and that draw from a world-wide resource 
of 3D-printing designs. At this time serious commercial business to business depositories of 3D-printing designs do not exist and the business model is still in its infancy. Many issues remain to be solved in the (digital) collaboration between the original equipment manufacturers to whom the 3D-printing designs belong to, the secure distribution and pricing of designs, and the (trusted) network of 3D-printing resources that can service clients globally.

\section{REFERENCES}

1. S. H. Huang, et al., "Additive manufacturing and its societal impact: a literature review," International journal of advanced manufacturing technology, vol. 67, pp. 1191-1203, 2013.

2. I. Campbell, et al., "Wohlers Report 2017 - Additive Manufacturing and 3D Printing State of the Industry Annual Worldwide Progress Report," Wohlers Associates Inc. , Fort Collins, Colorado 2017.

3. A. A. Zadpoor and J. Malda, "Additive Manufacturing of Biomaterials, Tissues, and Organs," Annals of biomedical engineering, vol. 45, pp. 1-11, 2017.

4. H. e. a. Wang, "Global, regional, and national life expectancy, all-cause mortality, and cause-specific mortality for 249 causes of death, 1980-2015: a systematic analysis for the Global Burden of Disease Study 2015," Lancet, vol. 388, pp. 1459-1544, 2016.

5. P. B. Sick, et al., "Initial Worldwide Experience With the WATCHMAN Left Atrial Appendage System for Stroke Prevention in Atrial Fibrillation," Journal of American college of cardiology, vol. 49, pp. 1490-1495, 2007.

6. A. Persona, et al., "Remote control and maintenance outsourcing networks and its applications in supply chain management," Journal of operations management, vol. 25, pp. 1275-1291, 2007.

7. J. Savolainen and M. Collan, "Additive manufacturing technology and business model change - a review of literature," Additive manufacturing, Vol. 32, Art. 101070, 2020.

8. M. Perini, et al., "Additive manufacturing for repairing: From damage identification and modeling to DLD," Rapid Prototyping Journal, 2020 (ahead of print).

9. C. Weller, et al., "Economic implications of 3D printing: Market structure models in light of additive manufacturing revisited," International Journal of Production Economics, vol. 164, pp. 43-56, 2015.

10. R. K. Mobley, An introduction to predictive maintenance, 2nd ed. Oxford, UK: Butterworth-Heinemann, 2002.

11. D. Thomas, "Costs, benefits, and adoption of additive manufacturing: a supply chain perspective," International journal of advanced manufacturing technology, vol. 85, pp. 1857-1876, 2016. 
Open Access This chapter is licensed under the terms of the Creative Commons Attribution 4.0 International License (http://creativecommons.org/licenses/ by $/ 4.0 /$ ), which permits use, sharing, adaptation, distribution and reproduction in any medium or format, as long as you give appropriate credit to the original author(s) and the source, provide a link to the Creative Commons licence and indicate if changes were made.

The images or other third party material in this chapter are included in the chapter's Creative Commons licence, unless indicated otherwise in a credit line to the material. If material is not included in the chapter's Creative Commons licence and your intended use is not permitted by statutory regulation or exceeds the permitted use, you will need to obtain permission directly from the copyright holder.

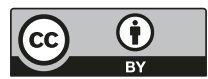

